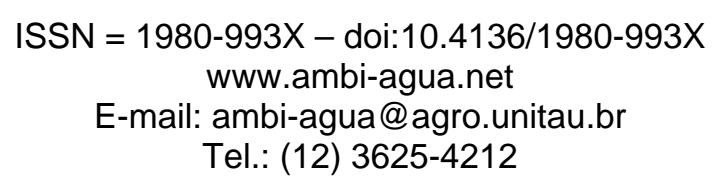

\title{
Análise de incerteza em um modelo matemático de qualidade da água aplicado ao Ribeirão do Ouro, Araraquara, SP, Brasil.
}

\author{
(http://dx.doi.org/10.4136/ambi-agua.196) \\ Daniel Jadyr Leite Costa'; ${ }^{\text {; Denilson Teixeira }}{ }^{2}$ \\ ${ }^{1}$ Mestre e Doutorando em Engenharia Hidráulica e Saneamento EESC/USP \\ e-mail: danielcosta.geo@gmail.com \\ ${ }^{2}$ Professor e Pesquisador do Programa de Mestrado em Desenvolvimento \\ Regional e Meio Ambiente - UNIARA - Brasil \\ e-mail: dteixeira@uniara.com.br
}

\section{RESUMO}

Os modelos matemáticos de qualidade de águas superficiais constituem-se em importante instrumento para auxiliar a tomada de decisão voltada tanto para a prevenção como para a correção da degradação dos recursos hídricos. No entanto, existem incertezas na determinação de parâmetros e variáveis de entrada dos modelos que podem alterar de forma significativa os resultados esperados. Se essas incertezas forem simplesmente ignoradas, podem ser tomadas decisões equivocadas, apoiadas em resultados de um modelo com pouca confiabilidade. Nesse contexto, o objetivo deste trabalho foi realizar uma análise de incerteza, a partir da utilização de um método estocástico sobre um modelo determinístico de qualidade de águas superficiais aplicado no Ribeirão do Ouro, localizado no município de Araraquara, SP. Foi utilizado o método de Análise de Erros de Primeira Ordem e os resultados, interpretados com relação ao atendimento aos padrões de qualidade de águas presentes na resolução CONAMA $357 / 2005$, subsidiaram a elaboração de mapas de qualidade das águas superficiais da bacia hidrográfica. Verificou-se significativa influência das atividades antrópicas sobre a qualidade das águas superficiais, havendo infração ambiental por parte do poluidor em um percurso com mais de $7 \mathrm{~km}$ de extensão. Por considerar as componentes de incertezas, o método estocástico demonstrou ser relevante para o gerenciamento dos recursos hídricos, pois possibilitou a apresentação dos resultados em termos de probabilidade de ocorrência.

Palavras-chave: Métodos estocásticos; modelagem matemática; águas superficiais; gestão de recursos hídricos.

\section{Uncertainty analysis in a water quality mathematical model applied to the Ribeirão do Ouro, Araraquara, SP, Brazil}

\begin{abstract}
Mathematical models for surface water quality constitute an important tool to assist decision-making used to prevent and to design water resources degradation restoration measures. However, there are uncertainties in the determination of parameters and input variables of the models that can significantly alter the expected results. If these uncertainties are simply ignored, wrong decisions can be taken, based on results of a low confidence level model. In this context, the aim of this study was to analyze the uncertainty, based on the use of a stochastic method, of a deterministic model of surface water quality applied to Ribeirão do Ouro, located in the Araraquara municipality, Brazil. The method of Analysis of First Order Errors was used and the results were interpreted taking into consideration the water quality standards recommended by CONAMA 357/2005 resolution to generate surface water
\end{abstract}


COSTA, D. J. L.; TEIXEIRA, D. Análise de incerteza em um modelo matemático de qualidade da água aplicado ao Ribeirão do Ouro, Araraquara, SP, Brasil. Ambi-Agua, Taubaté, v. 6, n. 2, p. 232-245, 2011. (doi:10.4136/ambi-agua.196)

quality maps for the watershed. Significant influence of human activities on the quality of surface water was observed in more than $7 \mathrm{~km}$ along the river indicating environmental violations by the polluter. Considering the uncertainty components, the stochastic method has proved to be relevant to the management of water resources, allowing the presentation of results in terms of probability of occurrence.

Keywords: Stochastic methods; mathematical modeling; surface water; water resource management.

\section{INTRODUÇÃO}

O atual modelo de desenvolvimento socioeconômico, o crescimento populacional acelerado e a necessidade de atendimento às diversas atividades humanas têm como resultado um panorama complexo em relação à degradação dos recursos naturais, especialmente evidenciado nos aspectos qualitativos e quantitativos dos recursos hídricos. Esse cenário tornou ainda mais importante os estudos e os procedimentos de gestão integrada desses recursos. Assim, cada vez mais, são necessárias ferramentas que quantifiquem direta ou indiretamente impactos ambientais, como passo inicial para a tomada de decisões que estejam direcionadas à manutenção e à recuperação da qualidade desses sistemas.

A Política Nacional de Recursos Hídricos, criada em 1997, define como instrumentos para alcance de seus objetivos o plano de recursos hídricos; o enquadramento dos corpos de água em classes, segundo seus usos preponderantes; a outorga e a cobrança pelo uso das águas; e o sistema de informações para o gerenciamento integrado dos recursos hídricos.

De acordo com Braga (2002), os instrumentos de políticas públicas de recursos hídricos são potencialmente indutores de conservação e recuperação desses recursos. Assim, é importante a utilização de ferramentas que possam subsidiar o adequado uso e acompanhamento da aplicabilidade desses instrumentos, de modo a integrar as inúmeras variáveis que formam o sistema em estudo.

O enfoque preventivo do controle ambiental tornou-se necessário para prever e avaliar os efeitos sobre o meio ambiente gerados por ações antrópicas. Dentro desse contexto, surgem os modelos matemáticos de qualidade hídrica, constituindo-se num importante instrumento para auxiliar a tomada de decisão tanto na prevenção como na correção da degradação dos recursos hídricos (Lima, 1997).

Para Jesus (2006), a modelagem matemática de qualidade da água é uma técnica que tem mostrado excelentes resultados na elaboração de prognósticos da qualidade da água em corpos hídricos, em função de distintos cenários de intervenções numa bacia hidrográfica.

A avaliação da qualidade ambiental para alocação de empreendimentos potencialmente geradores de poluição hídrica, a definição de respectivos níveis de controle para fontes existentes a instalarem-se em uma bacia hidrográfica para atendimento aos padrões de qualidade, a delimitação de trechos críticos de poluição e a previsão das alterações da qualidade da água ao longo do tempo e do espaço decorrentes de descargas acidentais são exemplos da ampla aplicação da modelagem matemática no gerenciamento dos recursos hídricos (Lima, 1997).

No entanto, existem restrições que devem ser consideradas quando se faz o uso de modelos matemáticos de uma forma geral. Os modelos matemáticos de qualidade de águas devem ser ajustados para que os valores de parâmetros e variáveis de entrada forneçam os resultados que melhor representem os valores observados de concentrações de poluentes no curso de água. Assim, face às imprecisões a que estão sujeitos os resultados obtidos a partir do ajuste, há a necessidade de implementar métodos que permitam a avaliação das incertezas nos parâmetros e variáveis de entrada.

Entre os componentes responsáveis pela introdução de incerteza nos dados de entrada de um modelo, podem-se citar os seguintes: erros na estimativa de um dado de entrada, baseado em valores de literatura, experiência pessoal, dentre outros; erros na amostragem; erros na 
COSTA, D. J. L.; TEIXEIRA, D. Análise de incerteza em um modelo matemático de qualidade da água aplicado ao Ribeirão do Ouro, Araraquara, SP, Brasil. Ambi-Agua, Taubaté, v. 6, n. 2, p. 232-245, 2011. (doi:10.4136/ambi-agua.196)

medição, calibração ou análise de laboratório; erros na transcrição ou transferência de resultados de análise ou de medições; e erros na estimativa de dados de entrada futuros, no caso de um modelo simular condições futuras (Von Sperling, 2007).

Desta forma, pode-se observar que, mesmo dados tradicionalmente não questionados em um modelo (como medições e resultados de análise) estão sujeitos, em maior ou menor grau, a um componente de incerteza. No entanto, a variabilidade no dado de entrada pode ser incorporada à interpretação dos resultados do modelo através da Análise de Incerteza, a qual permite a obtenção dos resultados expressos em termos probabilísticos, e não como simples valores únicos determinísticos (Von Sperling, 2007).

Sendo assim, o uso de modelos determinísticos como o QUAL 2E, QUAL 2K, MIKE 11, QUAL-UFMG, dentre outros, pode ser restringido quando se deseja trabalhar com o conceito de incerteza. Nesses casos, torna-se interessante seu uso conjunto com modelos que utilizam métodos estocásticos para resolução das equações, sendo exemplos desses modelos o SIMCAT - Simulation Catchment (Crabtree et al., 2006; Daldorph et al., 2001), o TOMCAT - Temporal/Overall Model for Catchments (Kannel et al., 2010; Kinniburgh et al., 1997) e o QUAL2E-UNCAS (Paliwal et al., 2007; Barnwell Jr. et al., 2004), cuja entrada de dados é uma distribuição estatística de probabilidades. Tais modelos produzem resultados como médias, percentis e intervalos de confiança para os trechos de rios simulados e permitem a análise de incertezas sobre os dados de entrada e suas previsões.

A partir dessas considerações, tem-se como objetivo no presente trabalho realizar uma análise de incerteza sobre um modelo determinístico de qualidade de águas superficiais, aplicado por Costa e Teixeira (2010) no ribeirão do Ouro, localizado no município de Araraquara, SP.

\section{MATERIAL E MÉTODOS}

\section{1. Área de estudo}

A bacia hidrográfica do ribeirão do Ouro pertence à Unidade de Gerenciamento de Recursos Hídricos Tietê-Jacaré (UGRHI 13) e localiza-se na porção central do estado de São Paulo, no município de Araraquara (SP). Possui, aproximadamente, $84,3 \mathrm{~km}^{2}$ e abrange em torno de $45 \%$ da área urbana desse município (Figura 1 ).

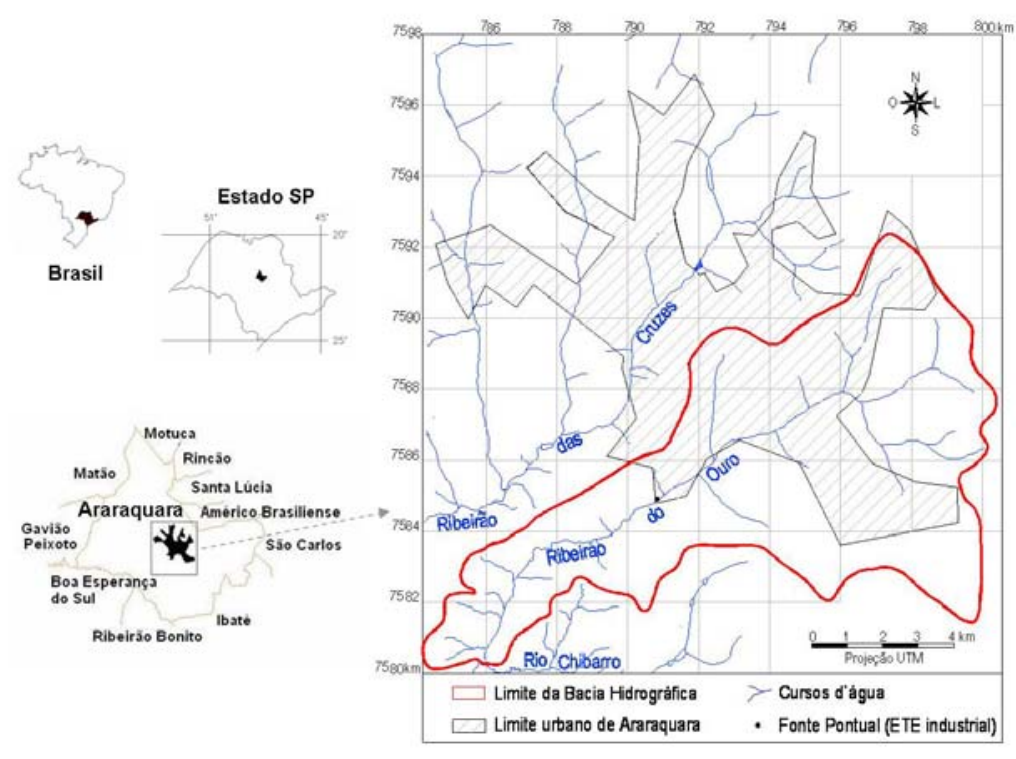

Figura 1. Localização da bacia hidrográfica do ribeirão do Ouro.

De acordo com Fuller (2008), pela classificação Koppen, essa bacia está localizada em uma região de clima Tropical de Altitude CWA, caracterizada por duas estações bem 
COSTA, D. J. L.; TEIXEIRA, D. Análise de incerteza em um modelo matemático de qualidade da água aplicado ao Ribeirão do Ouro, Araraquara, SP, Brasil. Ambi-Agua, Taubaté, v. 6, n. 2, p. 232-245, 2011. (doi:10.4136/ambi-agua.196)

definidas: um verão com temperaturas altas (média de $31{ }^{\circ} \mathrm{C}$ ) e pluviosidade elevada e um inverno com temperaturas amenas e pluviosidade reduzida.

Um mapeamento do uso e ocupação do solo, realizado a partir de imagens de satélite e apresentado por Teixeira et al. (2008), identificou que a bacia hidrográfica do ribeirão do Ouro está ocupada predominantemente por áreas agrícolas e pastos, abrangendo uma área de $48,6 \mathrm{~km}^{2}$ (ou $57,6 \%$ da área total da bacia). As áreas urbanas ocupam $27,1 \% \mathrm{e}$ as áreas verdes (incluindo reservas legais e matas ciliares), apenas 13,3\%.

O ribeirão do Ouro possui uma extensão aproximada de 19.900 metros. Ao longo de seu percurso recebe o lançamento de algumas fontes poluidoras. O modelo determinístico considerado neste trabalho foi aplicado, por Costa e Teixeira (2010), para caracterizar o efeito desses lançamentos sobre a qualidade de suas águas. O trecho simulado inicia-se na seção transversal, localizada a 13.400 metros de sua foz (Figura 1).

$\mathrm{Na}$ simulação realizada, verificou-se a presença de uma significativa fonte pontual proveniente de uma indústria e, ainda, outras fontes pontuais lançadas direta e indiretamente no corpo de água. As fontes pontuais indiretas são advindas de tributários que possuem elevadas concentrações de matéria orgânica, provenientes do lançamento clandestino de esgotos na área urbana.

O ribeirão do Ouro está enquadrado na Classe 4, de acordo com o Decreto n. ${ }^{\circ} 10.755$, de 22 de novembro de 1977, que dispõe sobre o enquadramento dos corpos de água receptores do estado de São Paulo, conforme classificação prevista no Decreto n. $^{\circ} 8.468$, de 8 de setembro de 1976.

\subsection{Modelo determinístico}

O percurso compreendido nessa simulação tem uma extensão de 13.400 metros e foi dividido em cinco trechos, sendo eles (com relação à seção inicial): $1^{\underline{0}}$ ) 0-3.100 m; $2^{\underline{0}}$ ) 3.100-

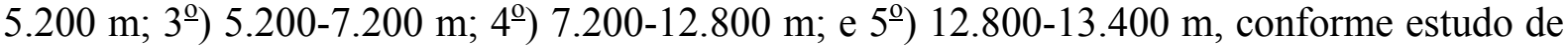
simulação previamente realizado por Costa e Teixeira (2010). Os critérios para a divisão de trechos estão baseados nas características hidráulicas do rio e na localização das fontes poluidoras. Para a simulação, os trechos foram divididos em elementos computacionais de comprimento $(\Delta \mathrm{x})$ igual a 100 metros. Na Figura 2, está apresentado o diagrama unifilar dos trechos simulados.

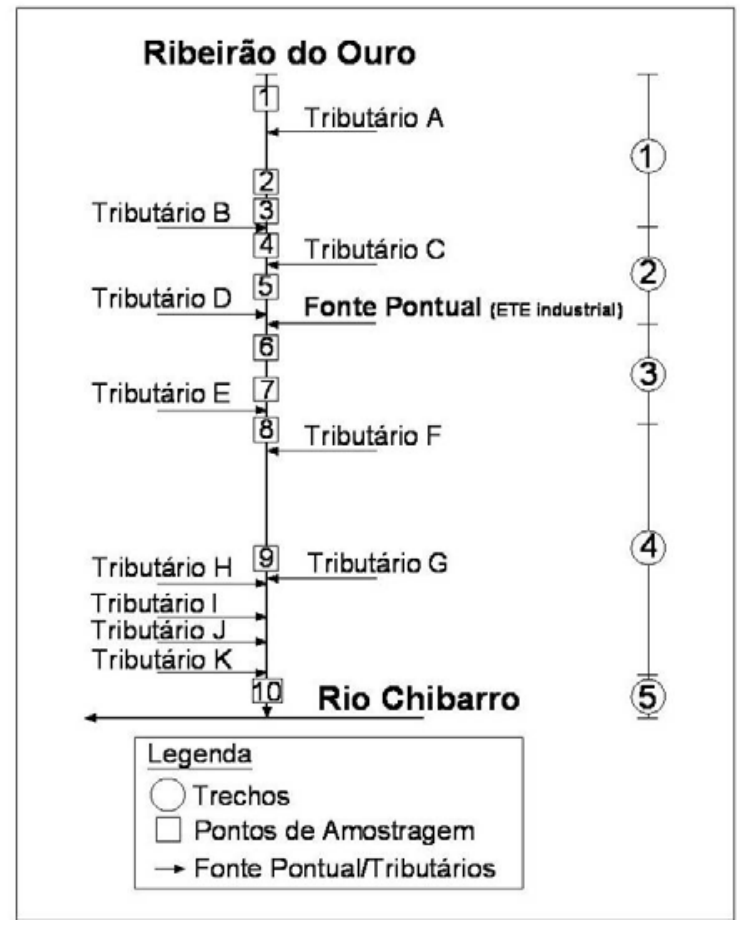

Figura 2. Diagrama unifilar dos trechos simulados do ribeirão do Ouro. 
COSTA, D. J. L.; TEIXEIRA, D. Análise de incerteza em um modelo matemático de qualidade da água aplicado ao Ribeirão do Ouro, Araraquara, SP, Brasil. Ambi-Agua, Taubaté, v. 6, n. 2, p. 232-245, 2011. (doi:10.4136/ambi-agua.196)

Os valores de Oxigênio Dissolvido (OD) e Demanda Bioquímica de Oxigênio (DBO) do ribeirão do Ouro, utilizados neste trabalho e no modelo aplicado por Costa e Teixeira (2010), encontram-se na Tabela 1.

Tabela 1. Valores de OD e DBO do Ribeirão do Ouro utilizados no modelo.

\begin{tabular}{l|c|c|c|c|c|c|c|c|c|c|c}
\hline \multirow{2}{*}{ Parâmetros } & \multicolumn{10}{c}{ Pontos de Amostragem } \\
\cline { 2 - 12 } & $\mathbf{1}$ & $\mathbf{2}$ & $\mathbf{3}$ & $\mathbf{4}$ & $\mathbf{5}$ & $\mathbf{6}$ & $\mathbf{7}$ & $\mathbf{8}$ & $\mathbf{9}$ & $\mathbf{1 0}$ & Padrão Classe 4 \\
\hline OD $[\mathrm{mg} / \mathrm{L}]$ & 7,0 & 6,2 & 6,6 & 5,0 & 5,4 & 3,9 & 1,9 & 0,8 & 1,5 & 0,8 & superior a $2,0 \mathrm{mg} / \mathrm{L}$ \\
\hline DBO $[\mathrm{mg} / \mathrm{L}]$ & 0,8 & 1,9 & 6,6 & 17,4 & 12,2 & 6,6 & 110,2 & 97,5 & 82,0 & 75,3 & - \\
\hline
\end{tabular}

A vazão calculada para a seção inicial do trecho simulado foi de $0,311 \mathrm{~m}^{3} / \mathrm{s}$. Os valores das vazões e concentrações de OD e DBO, para os tributários e efluente industrial, estão apresentados na Tabela 2.

Tabela 2. Vazões, OD e DBO dos tributários e efluente industrial.

\begin{tabular}{|c|c|c|c|c|c|c|c|c|c|c|c|c|}
\hline \multirow{2}{*}{ Parâmetros } & \multicolumn{11}{|c|}{ Tributários } & \multirow{2}{*}{$\begin{array}{c}\text { Fonte } \\
\text { Pontual } \\
\text { (ETE } \\
\text { industrial) } \\
\end{array}$} \\
\hline & A & B & C & D & $\mathbf{E}$ & $\mathbf{F}$ & G & $\mathbf{H}$ & I & $\mathbf{J}$ & $\mathbf{K}$ & \\
\hline Vazão [m³/s] & 0,037 & 0,043 & 0,058 & 0,004 & 0,004 & 0,008 & 0,01 & 0,008 & 0,006 & 0,01 & 0,013 & 0,1 \\
\hline $\mathrm{OD}[\mathrm{mg} / \mathrm{L}]$ & 5,0 & 0,0 & 5,0 & 5,0 & 5,0 & 5,0 & 5,0 & 5,0 & 5,0 & 5,0 & 5,0 & 0,0 \\
\hline $\mathrm{DBO}[\mathrm{mg} / \mathrm{L}]$ & 2,0 & 123,0 & 2,0 & 2,0 & 2,0 & 2,0 & 2,0 & 2,0 & 2,0 & 2,0 & 2,0 & 600,0 \\
\hline
\end{tabular}

O perfil de OD obtido encontra-se na Figura 3.

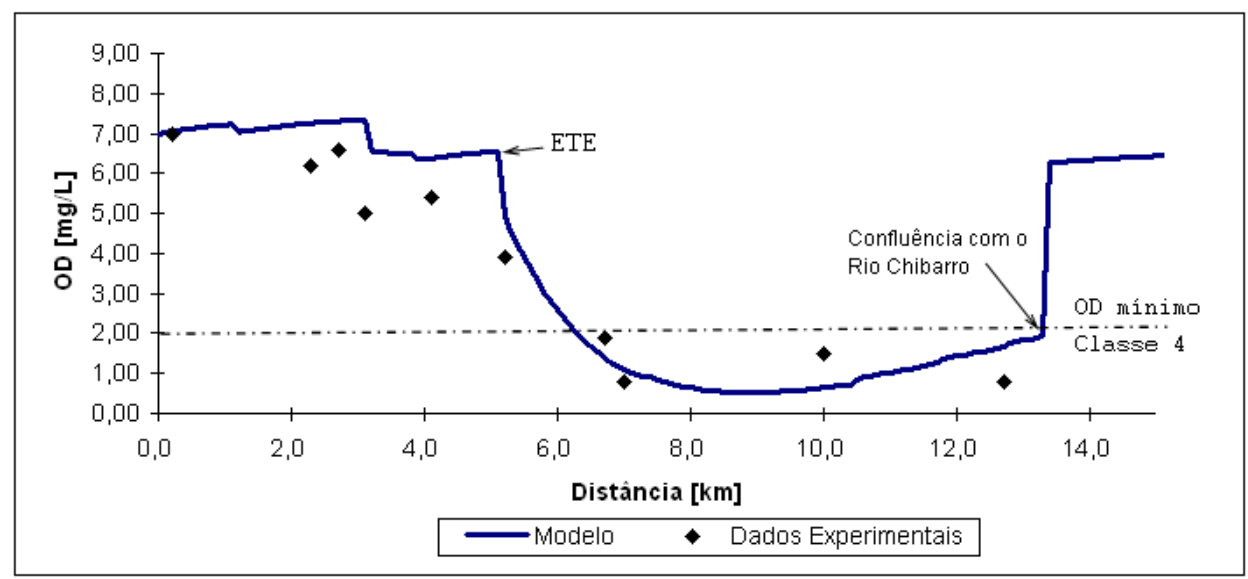

Figura 3. Perfil de OD para os trechos simulados do ribeirão do Ouro.

Fonte: Costa e Teixeira (2010).

\subsection{Avaliação de incertezas}

Em face às grandes incertezas a que estão sujeitos os resultados obtidos a partir do ajuste realizado no modelo determinístico, houve a necessidade de implementar no modelo um algoritmo que permita a avaliação das incertezas dos parâmetros e variáveis de entrada que foram utilizados.

O método estocástico para avaliação de incertezas utilizado neste trabalho se constitui na execução de 6 (seis) etapas, as quais estão descritas sucintamente abaixo. Uma referência de seu uso é o trabalho de Larentis (2004). 
COSTA, D. J. L.; TEIXEIRA, D. Análise de incerteza em um modelo matemático de qualidade da água aplicado ao Ribeirão do Ouro, Araraquara, SP, Brasil. Ambi-Agua, Taubaté, v. 6, n. 2, p. 232-245, 2011. (doi:10.4136/ambi-agua.196)

\subsubsection{Seleção das principais (mais sensíveis) fontes de incertezas}

Esta etapa consiste na aplicação de uma técnica de Análise de Sensibilidade para verificação dos parâmetros e variáveis mais sensíveis do modelo. Foi utilizada a técnica de Análise de Erros de Primeira Ordem, utilizando-se coeficientes normalizados de sensibilidade, os quais representam a porcentagem de variação na variável de saída resultante de uma variação de $80 \%$ em cada variável de entrada. A variável de saída analisada foi o OD mínimo, que representa a mínima concentração de oxigênio dissolvido obtida a partir das condições iniciais do modelo. Apenas como via de comparação, deve-se notar que o modelo QUAL2E-UNCAS ${ }^{1}$ também realiza a Análise de Sensibilidade a partir da técnica de Análise de Erros de Primeira Ordem.

\subsubsection{Definição de um intervalo de variação das fontes de incerteza}

Determinadas as variáveis mais sensíveis na etapa anterior, deve-se definir um intervalo de variação destas . Os intervalos a serem adotados podem considerar inúmeros aspectos, como: dados da literatura, nível de credibilidade dos métodos de análises utilizados (laboratoriais e numéricos), simulações realizadas com o próprio modelo e bom senso devido à experiência do pesquisador.

\subsubsection{Definição da distribuição de probabilidades}

Para a geração aleatória dos dados de entrada, duas distribuições de frequência usualmente utilizadas são a uniforme e a normal. Utilizou-se neste trabalho a distribuição uniforme, em que os valores variam dentro de uma faixa situada entre o valor mínimo e o valor máximo, com igual probabilidade de ocorrência em qualquer valor dentro da faixa.

\subsubsection{Geração de n números aleatórios para cada uma dessas variáveis dentro de seus respectivos intervalos}

Foram gerados 1.000 números aleatórios para cada uma das variáveis dentro de seus respectivos intervalos.

\subsubsection{Simulação da qualidade da água com os $\mathbf{n}$ conjuntos de valores de cada variável selecionada, gerados na etapa anterior}

Foi simulada a qualidade de água com os conjuntos de valores das variáveis selecionadas na etapa anterior a partir da equação analítica de Streeter e Phelps (1925) (Equação 1), para o trecho mais crítico da simulação realizada por Costa e Teixeira (2010), correspondente à seção de lançamento da ETE industrial até a confluência com o rio Chibarro.

$$
C_{t}=C_{s}-\left[\frac{K_{d} \cdot L_{0}}{K_{2}-K_{d}} \cdot\left(e^{-K_{d} \cdot t}-e^{-K_{2} \cdot t}\right)+\left(C_{s}-C_{0}\right) \cdot e^{-K_{2} \cdot t}\right]
$$

em que:

$\mathrm{C}_{\mathrm{t}}=$ concentração de OD em um instante de tempo $\mathrm{t}[\mathrm{mg} / \mathrm{L}]$

$\mathrm{C}_{\mathrm{s}}=$ concentração de saturação de $\mathrm{OD}[\mathrm{mg} / \mathrm{L}]$

$\mathrm{C}_{0}=$ concentração inicial de $\mathrm{OD}, \operatorname{logo}$ após a mistura $[\mathrm{mg} / \mathrm{L}]$

$\mathrm{K}_{\mathrm{d}}=$ coeficiente de desoxigenação no rio $\left[\mathrm{dia}^{-1}\right]$

$\mathrm{K}_{2}=$ coeficiente de reoxigenação $\left[\mathrm{dia}^{-1}\right]$

$\mathrm{L}_{0}=\mathrm{DBO}$ remanescente em $\mathrm{t}=0[\mathrm{mg} / \mathrm{L}]$

\footnotetext{
${ }^{1}$ O QUAL2E-UNCAS é o módulo de análise de incertezas do QUAL 2E, distribuído pela United States Environmental Protection Agency (USEPA).
} 
COSTA, D. J. L.; TEIXEIRA, D. Análise de incerteza em um modelo matemático de qualidade da água aplicado ao Ribeirão do Ouro, Araraquara, SP, Brasil. Ambi-Agua, Taubaté, v. 6, n. 2, p. 232-245, 2011. (doi:10.4136/ambi-agua.196)

\subsubsection{Obtenção dos valores esperados}

São obtidos os valores de concentração médios (valores esperados), máximos e mínimos no corpo de água, para os instantes de tempo utilizados na Equação 1.

\subsection{Interpretação e apresentação espacial dos resultados}

Com os 1.000 valores de OD mínimo obtidos, pode-se realizar a interpretação em termos de sua distribuição de frequência e a probabilidade de atendimento à legislação, considerando-se a classe em que o ribeirão está enquadrado.

Além dos resultados numéricos dos perfis de OD apresentados na Figura 4, estes podem ser apresentados de forma espacial (geográfica), a partir de uma carta georreferenciada e digitalizada da bacia hidrográfica em estudo, considerando-se os perfis de OD máximo, médio e mínimo (Figuras 7, 8 e 9). Foi utilizada uma carta digital do IBGE (1983), folha topográfica de Araraquara (SF-22-X-D-VI-4), com escala 1:50.000, sendo a mesma utilizada para a elaboração parcial da Figura 1.

\section{RESULTADOS E DISCUSSÃO}

\subsection{Avaliação de incertezas}

Com a realização da avaliação de incertezas a partir das seis etapas mencionadas, foi possível selecionar as principais (mais sensíveis) fontes de incertezas com base na variação percentual do OD mínimo (Análise de Erros de Primeira Ordem), devido à variação de $80 \%$ em torno do valor fixo de cada variável de entrada (Tabela 3).

Tabela 3. Variação percentual sobre o OD mínimo, a partir da Análise de Erros de Primeira Ordem.

\begin{tabular}{c|c|c|c|c}
\hline $\begin{array}{c}\text { Dado de } \\
\text { entrada }\end{array}$ & Unidade & $\begin{array}{c}\text { Valor } \\
\text { Fixo }\end{array}$ & $\begin{array}{c}\text { Percentual de } \\
\text { variação em torno do } \\
\text { valor fixo (\%) }\end{array}$ & $\begin{array}{c}\text { Variação percentual } \\
\text { sobre o OD mínimo } \\
\text { (\%) }\end{array}$ \\
\hline $\mathrm{Q}_{\mathrm{r}}$ & $\mathrm{m} / \mathrm{s}$ & 0,4527 & 80 & 28,8 \\
$\mathrm{Q}_{\mathrm{e}}$ & $\mathrm{m} / \mathrm{s}$ & 0,100 & 80 & 10,5 \\
$\mathrm{DBO}_{\mathrm{r}}$ & $\mathrm{mg} / \mathrm{L}$ & 11 & 80 & 0,4 \\
$\mathrm{DBO}_{\mathrm{e}}$ & $\mathrm{mg} / \mathrm{L}$ & 600 & 80 & 9,6 \\
$\mathrm{OD}_{\mathrm{r}}$ & $\mathrm{mg} / \mathrm{L}$ & 6,37 & 80 & 0,7 \\
$\mathrm{OD}_{\mathrm{e}}$ & $\mathrm{mg} / \mathrm{L}$ & 0 & 80 & 0 \\
$\mathrm{~K}_{1}$ & $1 / \mathrm{d}$ & 0,43 & 80 & 11,0 \\
$\mathrm{~K}_{\mathrm{d}}$ & $1 / \mathrm{d}$ & 0,77 & 80 & 9,0 \\
$\mathrm{~K}_{2}$ & $1 / \mathrm{d}$ & 15 & 80 & 21,3 \\
$\mathrm{OD}_{\mathrm{sat}}$ & $\mathrm{mg} / \mathrm{L}$ & 7,75 & 80 & 10,3 \\
$\mathrm{~d}$ & $\mathrm{~km}$ & 40 & 80 & 0 \\
$\mathrm{v}$ & $\mathrm{m} / \mathrm{s}$ & 0,2 & 80 & 0 \\
$\mathrm{E}_{\mathrm{DBO}}$ & $\%$ & 0 & 80 & 0 \\
\hline
\end{tabular}

Legenda:

$\mathrm{Q}_{\mathrm{r}}=$ vazão do rio; $\mathrm{Q}_{\mathrm{e}}=$ vazão do efluente

$\mathrm{DBO}_{\mathrm{r}}=\mathrm{DBO}$ do rio; $\mathrm{DBO}_{\mathrm{e}}=\mathrm{DBO}$ do efluente

$\mathrm{OD}_{\mathrm{r}}=$ concentração de $\mathrm{OD}$ do rio; $\mathrm{OD}_{\mathrm{e}}=$ concentração de $\mathrm{OD}$ do efluente

$\mathrm{K}_{1}=$ coeficiente de desoxigenação no laboratório; $\mathrm{K}_{\mathrm{d}}=$ coeficiente de desoxigenação

no rio; $\mathrm{K}_{2}=$ coeficiente de reoxigenação

$\mathrm{OD}_{\text {sat }}=$ concentração de saturação de $\mathrm{OD}$ no rio

$\mathrm{d}=$ distância do trecho simulado

$\mathrm{v}=$ velocidade media de escoamento

$\mathrm{E}_{\mathrm{DBO}}=$ eficiência de remoção de DBO na ETE 
COSTA, D. J. L.; TEIXEIRA, D. Análise de incerteza em um modelo matemático de qualidade da água aplicado ao Ribeirão do Ouro, Araraquara, SP, Brasil. Ambi-Agua, Taubaté, v. 6, n. 2, p. 232-245, 2011. (doi:10.4136/ambi-agua.196)

Em seguida, foram definidos os intervalos de variação de algumas variáveis (dados de entrada), consideradas como fontes de incertezas, do modelo determinístico utilizado por Costa e Teixeira (2010).

As variáveis que possuíram os intervalos de variação mais significativos foram: vazão do ribeirão do Ouro $\left(\mathrm{Q}_{\mathrm{r}}\right)$, vazão do efluente industrial $\left(\mathrm{Q}_{\mathrm{e}}\right)$, Demanda Bioquímica de Oxigênio $\left(\mathrm{DBO}_{\mathrm{e}}\right)$, e coeficiente de reoxigenação $\left(\mathrm{K}_{2}\right)$. A vazão do ribeirão do Ouro $\left(\mathrm{Q}_{\mathrm{r}}\right)$ possui incertezas numéricas, pois ela foi estimada a partir do método de regionalização hidrológica. As incertezas da vazão do efluente industrial $\left(\mathrm{Q}_{\mathrm{e}}\right)$ e de sua Demanda Bioquímica de Oxigênio $\left(\mathrm{DBO}_{\mathrm{e}}\right)$ justificam-se, pois seus valores foram estimados, respectivamente, a partir de dados da literatura e uso de bom senso. As variações em torno do valor fixo nos dados de entrada encontram-se na Tabela 4.

Tabela 4. Intervalo de variação (em percentagem) dos dados de entrada do modelo determinístico utilizado por Costa e Teixeira (2010).

\begin{tabular}{c|c|c|c|c|c}
\hline \multirow{2}{*}{$\begin{array}{c}\text { Dado de } \\
\text { entrada }\end{array}$} & \multirow{2}{*}{ Unidade } & \multirow{2}{*}{$\begin{array}{c}\text { Valor } \\
\text { Fixo }\end{array}$} & $\begin{array}{c}\text { Percentual de } \\
\text { variação em torno } \\
\text { do valor fixo (\%) }\end{array}$ & \multicolumn{2}{|c}{ Faixa de Valores } \\
\cline { 5 - 6 } & & & Valor Mínimo & Valor Máximo \\
\hline $\mathrm{Q}_{\mathrm{r}}$ & $\mathrm{m} / \mathrm{s}$ & 0,452 & 10 & 0,407 & 0,497 \\
$\mathrm{Q}_{\mathrm{e}}$ & $\mathrm{m} / \mathrm{s}$ & 0,100 & 20 & 0,080 & 0,120 \\
$\mathrm{DBO}_{\mathrm{r}}$ & $\mathrm{mg} / \mathrm{L}$ & 11,0 & 5 & 10,4 & 11,5 \\
$\mathrm{DBO}_{\mathrm{e}}$ & $\mathrm{mg} / \mathrm{L}$ & 600 & 20 & 480 & 720 \\
$\mathrm{OD}_{\mathrm{r}}$ & $\mathrm{mg} / \mathrm{L}$ & 6,4 & 5 & 6,0 & 6,7 \\
$\mathrm{OD}_{\mathrm{e}}$ & $\mathrm{mg} / \mathrm{L}$ & 0 & 0 & 0 & 0 \\
$\mathrm{~K}_{1}$ & $1 / \mathrm{d}$ & 0,43 & 5 & 0,40 & 0,45 \\
$\mathrm{~K}_{\mathrm{d}}$ & $1 / \mathrm{d}$ & 0,77 & 5 & 0,73 & 0,80 \\
$\mathrm{~K}_{2}$ & $1 / \mathrm{d}$ & 15 & 30 & 10,5 & 19,5 \\
$\mathrm{OD}_{\text {sat }}$ & $\mathrm{mg} / \mathrm{L}$ & 7,75 & 5 & 7,36 & 8,13 \\
$\mathrm{~d}$ & $\mathrm{~km}$ & 40 & 0 & 40 & 40 \\
$\mathrm{v}$ & $\mathrm{m} / \mathrm{s}$ & 0,2 & 5 & 0,19 & 0,21 \\
$\mathrm{E}_{\mathrm{DBO}}$ & $\%$ & 0 & 5 & 0 & 0 \\
\hline
\end{tabular}

A partir da Análise de Erros de Primeira Ordem, realizada na primeira etapa do método utilizado para avaliação de incertezas, verificou-se que o coeficiente de reoxigenação (K2) é a segunda variável à qual o modelo é mais sensível ( $\mathrm{Qr}$ foi a variável mais sensível). Além desta ser muito sensível para o modelo, a sua determinação é realizada por meio de equações empíricas presentes na literatura, que possuem grandes incertezas quando utilizadas em corpos de água diferentes daqueles em que foram coletados os dados experimentais. Esses dois aspectos justificaram a adoção de $30 \%$ de variação em torno de seu valor fixo.

Apenas por via de esclarecimento, o uso da técnica de traçadores gasosos para a determinação do coeficiente de reoxigenação (K2) pode reduzir o grau de incerteza dos valores obtidos, no entanto, Costa (1999) ressalta que além de onerosa, quando empregada em campo, essa técnica exige um grupo de profissionais especializados.

As incertezas de $5 \%$ de variação em torno do valor fixo presentes nas variáveis DBOr, ODr, K1, Kd, ODsat, v e EDBO se justificam em razão dos métodos utilizados na determinação de cada variável.

Após a realização das quatro etapas iniciais do método de avaliação de incertezas, foi simulada a qualidade de água, para o trecho mais crítico, com os conjuntos de valores das variáveis selecionadas na etapa anterior a partir a equação analítica de Streeter e Phelps (1925) (Equação 1). Assim, foram obtidos os valores de concentração máximos (Cenário 1), médios (valores esperados - Cenário 2) e mínimos (Cenário 3) no corpo de água, para os 
COSTA, D. J. L.; TEIXEIRA, D. Análise de incerteza em um modelo matemático de qualidade da água aplicado ao Ribeirão do Ouro, Araraquara, SP, Brasil. Ambi-Agua, Taubaté, v. 6, n. 2, p. 232-245, 2011. (doi:10.4136/ambi-agua.196)

instantes de tempo utilizados na Equação 1, os quais possibilitaram a obtenção dos perfis de OD apresentados na Figura 4.

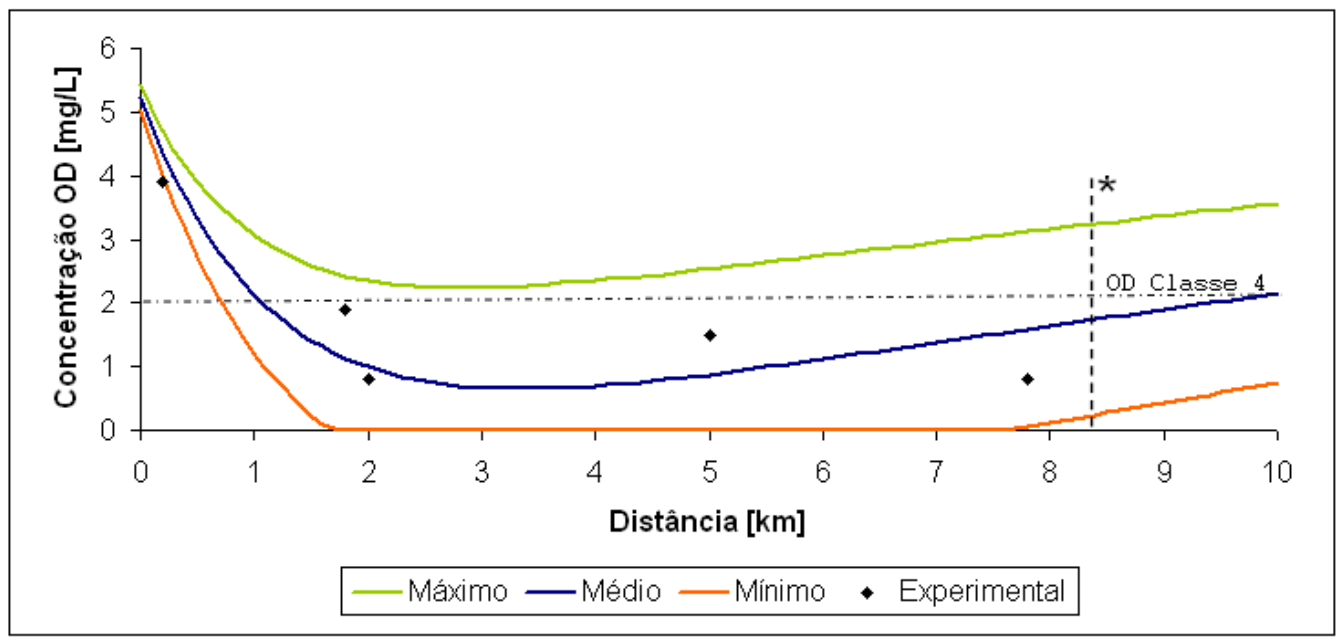

Figura 4. Perfis de OD máximo, médio e mínimo no trecho crítico.

* Localização da seção de confluência com o rio Chibarro. Isso significa que o perfil de OD a jusante desta seção é apenas representativo.

\subsection{Interpretação e apresentação espacial dos resultados}

Com os 1.000 valores de OD mínimo obtidos, faz-se a interpretação em termos de sua distribuição de frequência, que pode ser simples (Figura 5) ou acumulada (Figura 6).

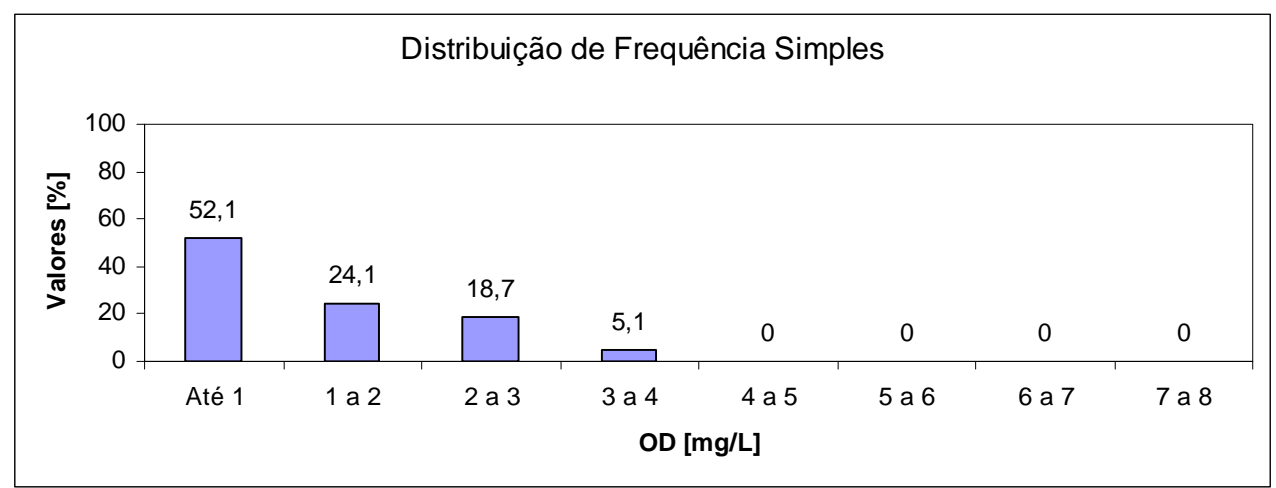

Figura 5. Distribuição de frequência simples de OD mínimo.

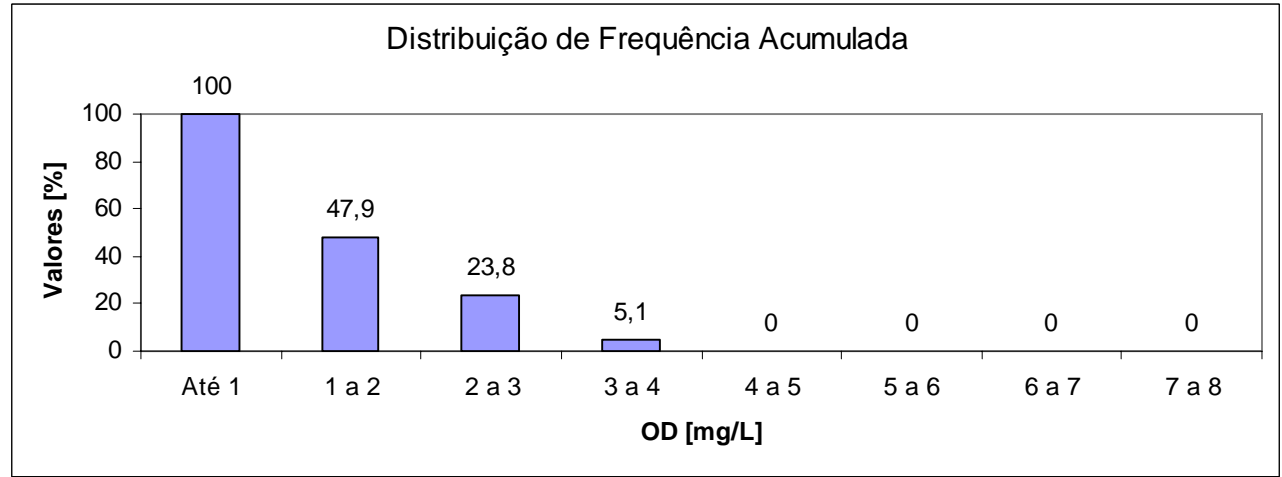

Figura 6. Distribuição de frequência acumulada de OD mínimo.

\subsubsection{Probabilidade de atendimento à legislação}

A partir da distribuição de frequência acumulada, pode-se verificar a probabilidade de atendimento à legislação, considerando-se a classe em que o ribeirão está enquadrado 
COSTA, D. J. L.; TEIXEIRA, D. Análise de incerteza em um modelo matemático de qualidade da água aplicado ao Ribeirão do Ouro, Araraquara, SP, Brasil. Ambi-Agua, Taubaté, v. 6, n. 2, p. 232-245, 2011. (doi:10.4136/ambi-agua.196)

(Tabela 5). Essa tabela mostra que a probabilidade de atendimento à legislação do ribeirão do Ouro para o evento simulado foi de $20,2 \%$.

Tabela 5. Probabilidade de atendimento à legislação.

\begin{tabular}{c|c|c}
\hline $\begin{array}{c}\text { Class } \\
\mathbf{e}\end{array}$ & $\begin{array}{c}\text { OD }^{*} \\
{[\mathbf{m g} / \mathbf{L}]}\end{array}$ & $\begin{array}{c}\text { Probabilidade de atendimento } \\
{[\%]}\end{array}$ \\
\hline $\mathbf{1}$ & $\geq \mathbf{6}$ & 0 \\
$\mathbf{2}$ & $\geq \mathbf{5}$ & 0 \\
$\mathbf{3}$ & $\geq \mathbf{4}$ & 0,3 \\
$\mathbf{4}$ & $\geq \mathbf{2}$ & 20,2 \\
\hline * Padrões de qualidade de águas em corpos receptores \\
\multicolumn{2}{l}{ estabelecidos na resolução CONAMA 357/2005 (Brasil, 2005). }
\end{tabular}

\subsubsection{Espacialização dos resultados obtidos}

Os resultados numéricos dos perfis de OD reproduzidos na Figura 4 estão apresentados de forma espacial (geográfica), a partir de uma carta georeferenciada e digitalizada da bacia hidrográfica em estudo, considerando-se os perfis de OD máximo (Cenário 1), médio (Cenário 2) e mínimo (Cenário 3), respectivamente, nas Figuras 7, 8 e 9.

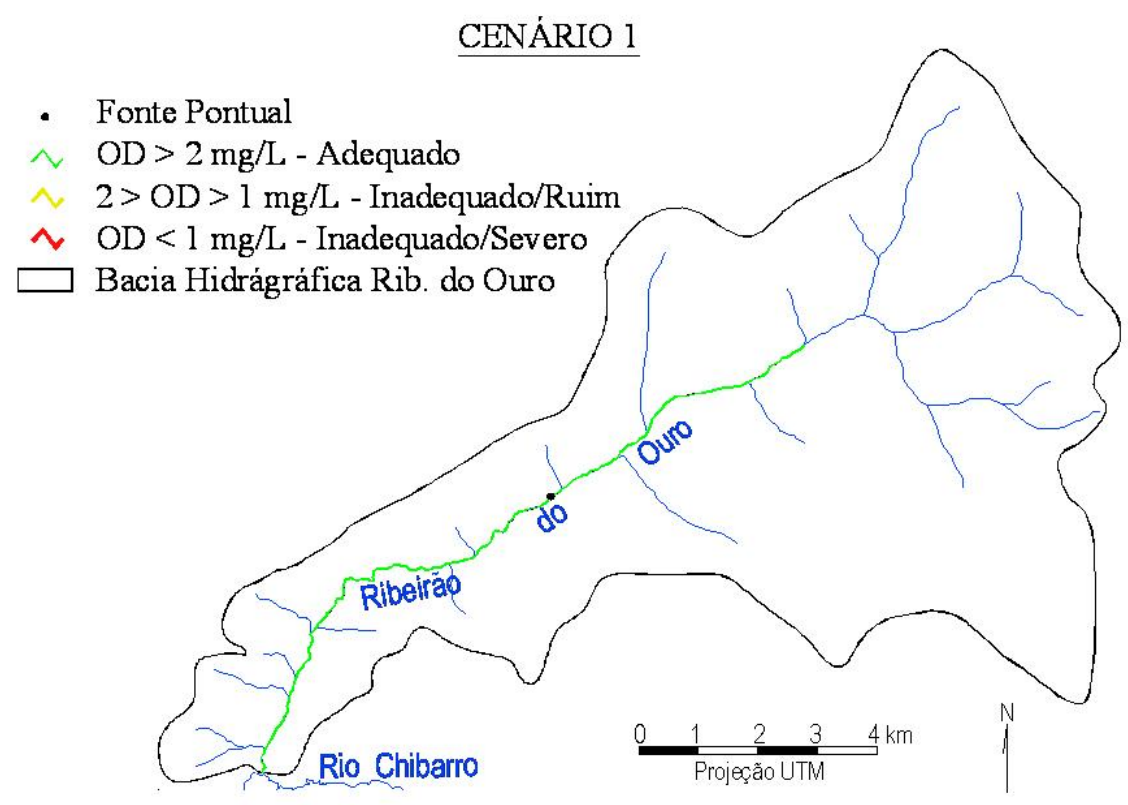

Figura 7. Espacialização geográfica do perfil máximo de OD (Cenário 1).

O Cenário 2, representado na Figura 8, apresenta a situação mais provável de ocorrência e possui um trecho do ribeirão, em torno de $1.900 \mathrm{~m}$, com concentração de OD abaixo de 1 $\mathrm{mg} / \mathrm{L}$. Pode-se considerar que no trecho há o desenvolvimento de um quadro de poluição severa, com excessiva depleção da concentração natural de OD, o qual pode trazer consequências negativas para a biota aquática.

As Figuras 7 e 9 apresentam os cenários ambientais extremos (Cenários 1 e 3, respectivamente), os quais possuem baixa probabilidade de ocorrência. O Cenário 1 seria o mais desejável, e pode ser considerado adequado para um corpo de água que esteja enquadrado na Classe 4, como é o caso do ribeirão do Ouro. Afinal, os níveis de concentração de OD encontram-se acima do padrão de qualidade estabelecido na legislação vigente (Brasil, 2005). O Cenário 3 representa a situação mais crítica, possuindo um trecho com distância longitudinal em torno de $6.950 \mathrm{~m}$, com concentração de OD abaixo de $1 \mathrm{mg} / \mathrm{L}$. A ocorrência de baixas concentrações de OD pode acarretar um quadro de poluição severa e, em termos 
COSTA, D. J. L.; TEIXEIRA, D. Análise de incerteza em um modelo matemático de qualidade da água aplicado ao Ribeirão do Ouro, Araraquara, SP, Brasil. Ambi-Agua, Taubaté, v. 6, n. 2, p. 232-245, 2011. (doi:10.4136/ambi-agua.196)

comparativos, apresentar um quadro de poluição ainda mais intenso que o trecho mais crítico presente no Cenário 2 (trecho classificado como Inadequado/Severo), devido a sua maior extensão longitudinal.

\section{CENÁRIO 2}

- Fonte Pontual

$\curvearrowright \mathrm{OD}>2 \mathrm{mg} / \mathrm{L}-$ Adequado $2>\mathrm{OD}>1 \mathrm{mg} / \mathrm{L}$ - Inadequado/Ruim

$\approx$ OD $<1 \mathrm{mg} / \mathrm{L}$ - Inadequado/Severo

Bacia Hidrágráfica Rib. do Ouro

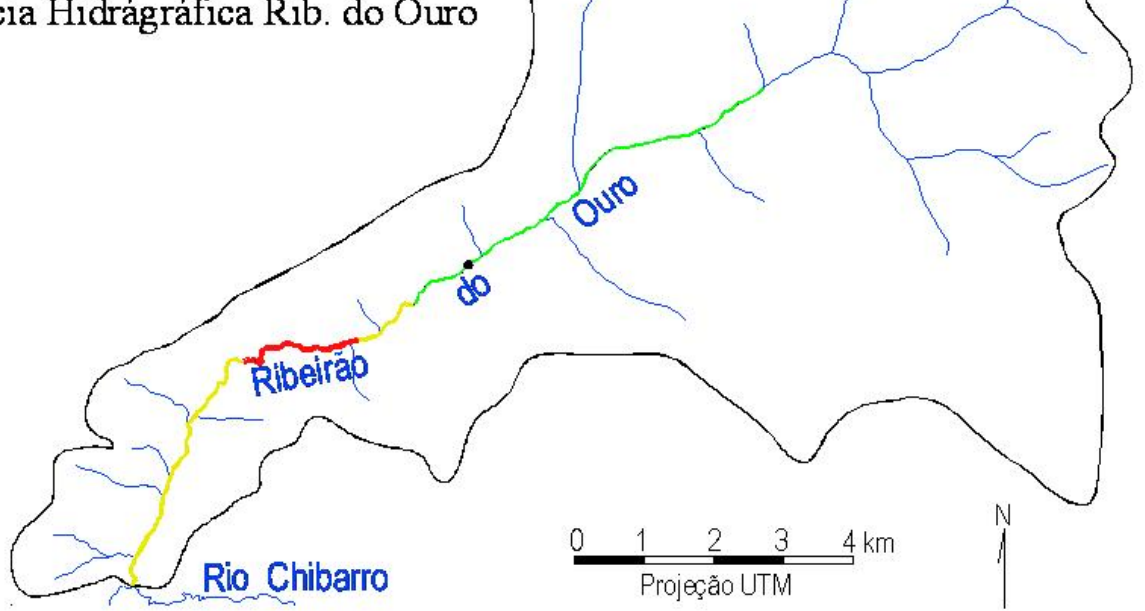

Espacialização geográfica do perfil médio de OD (Cenário 2).

Figura 8.

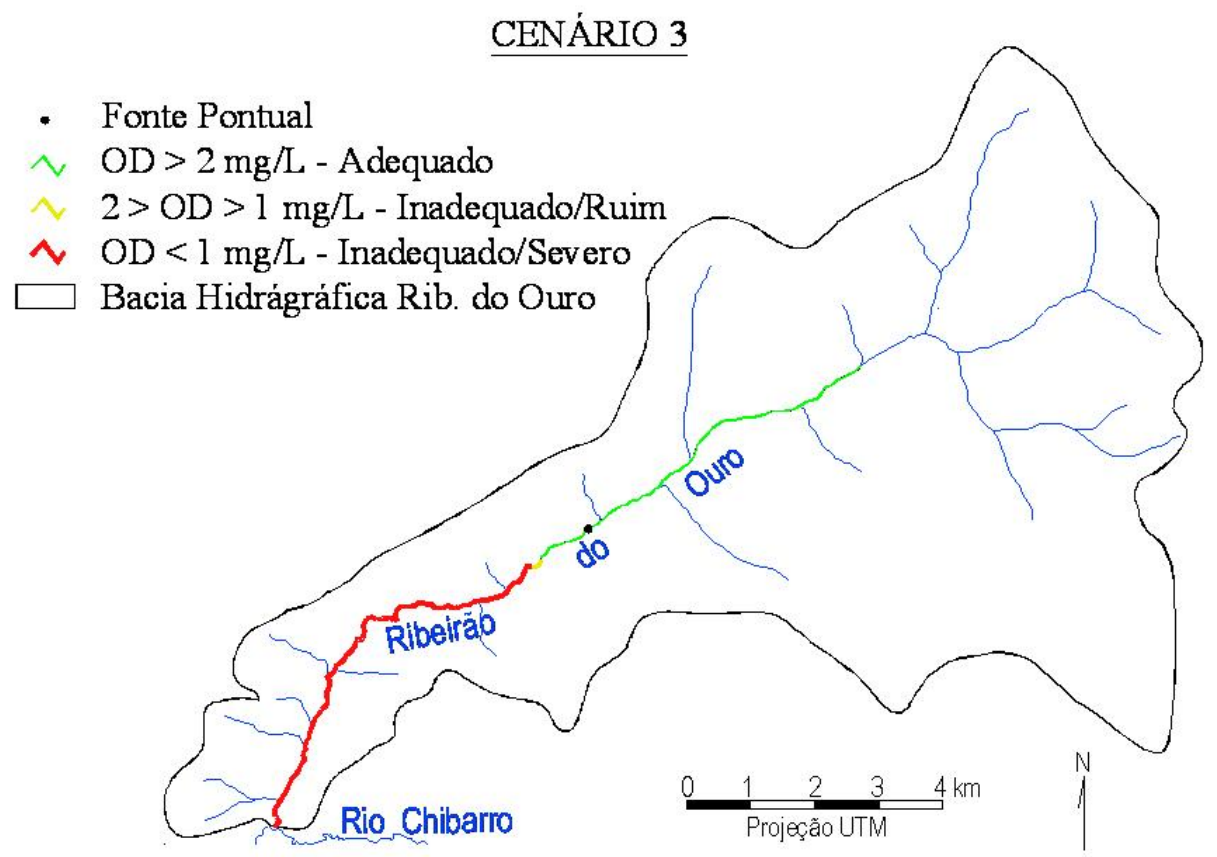

Figura 9. Espacialização geográfica do perfil mínimo de OD (Cenário 3).

A representação dos resultados numéricos na forma de mapas de qualidade das águas, como os apresentados nas Figuras 7, 8 e 9, constitui-se na mais completa forma de interação entre o pesquisador e os tomadores de decisão. Esse tipo de informação é muito importante para subsidiar o processo de gestão integrada dos recursos hídricos. Permite, também - o que pode ser de interesse principalmente da academia -, a definição de estudos e pesquisas 
COSTA, D. J. L.; TEIXEIRA, D. Análise de incerteza em um modelo matemático de qualidade da água aplicado ao Ribeirão do Ouro, Araraquara, SP, Brasil. Ambi-Agua, Taubaté, v. 6, n. 2, p. 232-245, 2011. (doi:10.4136/ambi-agua.196)

voltadas aos seguintes temas: toxicologia, análise de risco, avaliação de impactos ambientais, indicadores biológicos de qualidade de água, entre outros. Cabe ainda destacar o potencial dessa ferramenta como subsídio para o estabelecimento de programas de monitoramento de qualidade de água para a bacia hidrográfica em questão.

\section{CONCLUSÃO}

Verificou-se que as atividades antrópicas presentes na bacia hidrográfica do ribeirão do Ouro exercem significativa influência sobre a qualidade das águas superficiais. Considerandose o perfil médio de OD do evento simulado, as concentrações de OD encontram-se predominantemente abaixo do limite fixado para corpos de água enquadrados como Classe 4 na Resolução 357/2005 do CONAMA, havendo infração ambiental por parte do poluidor em um percurso com mais de $7 \mathrm{~km}$ de extensão.

Como os processos ambientais e os lançamentos de cargas poluidoras são dinâmicos (podendo ser classificados como nãopermanentes), justifica-se a necessidade de campanhas de amostragens contínuas, por meio de um eficiente monitoramento das características qualiquantitativas do corpo receptor e das cargas poluidoras pontuais e difusas. Os dados obtidos poderão subsidiar a validação de modelos matemáticos de qualidade de águas superficiais e a construção de um sistema de indicadores de qualidade ambiental.

O uso do método estocástico para a análise de incertezas sobre o modelo determinístico mostrou ser de fundamental importância para subsidiar o gerenciamento dos recursos hídricos da bacia hidrográfica do ribeirão do Ouro, principalmente por facilitar a interpretação dos resultados por parte dos gestores e tomadores de decisão. Ao considerar os componentes de incerteza presentes nos valores numéricos, a interpretação dos resultados passa a ser realizada de forma mais real, pois estes passam a ser expressos em termos de probabilidade de ocorrência e não como simples valores únicos ou determinísticos.

\section{REFERENCIAS}

BARNWELL JR., T. O.; BROWN, L. C.; WHITTEMORE, R. C. Importance of field data in stream water quality modeling using QUAL2E-UNCAS. Journal of Environmental Engineering, v. 130, n. 6, p. 643-647, 2004. http://dx.doi.org/10.1061/(ASCE)0733-9372(2004)130:6(643)

BRAGA, B. et al. Introdução à engenharia ambiental. São Paulo: Prentice Hall, 2002.

BRASIL. Lei $\mathrm{n}^{\circ}$ 9.433, de 8 de janeiro de 1997. Institui a Política Nacional de Recursos Hídricos. Lex: Código de Águas: e legislação correlata. Brasília: Senado Federal, Subsecretaria de Edições Técnicas, 2003. 234 p. (Coleção Ambiental; 1)

BRASIL. Ministério do Meio Ambiente. Conselho Nacional do Meio Ambiente. (CONAMA). Resolução $\mathbf{n}^{\circ}$ 357, de 17 de março de 2005. Dispõe sobre as classificações dos corpos de água e diretrizes ambientais para o seu enquadramento, bem como estabelece as condições e padrões de lançamento de efluentes, e dá outras providências. Disponível em: <www.mma.gov.br/port/conama/res/res05/res35705.pdf>. Acesso em: 12 nov. 2010.

COSTA, D. J. L.; TEIXEIRA, D. Aplicação de modelo de autodepuração para avaliação da qualidade da água do Ribeirão do Ouro, Araraquara, SP. Revista Uniara, v. 13, n. 1, p. 49-62, 2010. 
COSTA, D. J. L.; TEIXEIRA, D. Análise de incerteza em um modelo matemático de qualidade da água aplicado ao Ribeirão do Ouro, Araraquara, SP, Brasil. Ambi-Agua, Taubaté, v. 6, n. 2, p. 232-245, 2011. (doi:10.4136/ambi-agua.196)

COSTA, O. S. Contribuição à metodologia para determinação indireta do coeficiente de reaeração dos escoamentos naturais de água com emprego do método da sonda solúvel. 1999. 156f. Tese (Doutorado em Hidráulica e Saneamento) - Escola de Engenharia de São Carlos, Universidade de São Paulo, São Carlos, 1999.

CRABTREE, B.; SEWARD, A. J.; THOMPSON, L. A case study of regional catchment water quality modelling to identify pollution control requirements. Water Science and Technology, v. 53, n. 10, p. 47-54, 2006. http://dx.doi.org/10.2166/wst.2006.296

DALDORPH, P. W. G.; SPRAGGS, G. E.; LEES, M. J.; WHEATER, H. S.; CHAPRA, S. C. Integrated lake and catchment phosphorus model - A eutrophication management tool. II: Application to Rutland Water. Journal of the Chartered Institution of Water and Environmental Management, v. 15, n. 3, p. 182-189, 2001. http://dx.doi.org/10.1111/j.1747-6593.2001.tb00330.x

FULLER, B. B. Caracterização espaço-temporal dos recursos hídricos superficiais da sub-bacia do ribeirão do Ouro, Araraquara, SP. 2008. 157f. Dissertação (Mestrado em Desenvolvimento Regional e Meio Ambiente) - Centro Universitário de Araraquara, Araraquara, 2008.

INSTITUTO BRASILEIRO DE GEOGRAFIA E ESTATÍSTICA. Carta do Brasil. Folha topográfica de Araraquara (SF-22-X-D-VI-4). Rio de Janeiro: IBGE, 1983. Escala $1: 50.000$.

JESUS, J. A. O. Utilização de modelagem matemática 3D na gestão da qualidade da água em mananciais - aplicação no reservatório Billings. 2006. 142f. Tese (Doutorado em Saúde Pública) - Faculdade de Saúde Pública, Universidade de São Paulo, São Paulo, 2006.

KANNEL, P. R.; KANEL, S. R.; LEE, S.; LEE, Y. S.; GAN, T. Y. A review of public domain water quality models for simulating dissolved oxygen in rivers and streams. Environmental Modeling and Assessment, v. 15, n.1, p. 1-22, 2010.

KINNIBURGH, J. H.; TINSLEY, M. R.; BENNETT, J. Orthophosphate concentrations in the River Thames. Journal of the Chartered Institution of Water and Environmental Management, v. 11, n. 3, p. 178-185, 1997. http://dx.doi.org/10.1111/j.17476593.1997.tb00113.x

LARENTIS, D. G. Modelagem matemática da qualidade da água em grandes bacias: Sistema Taquari-Antas, RS. 2004. 159f. Dissertação (Mestrado em Recursos Hídricos e Saneamento Ambiental) - Instituto de Pesquisas Hidráulicas, Universidade Federal do Rio Grande do Sul, Porto Alegre, 2004.

LIMA, C. A. A. O uso do modelo QUAL2E na simulação da qualidade das águas do Rio Jacaré-Guaçu. 1997. 179f. Dissertação (Mestrado em Hidráulica e Saneamento) Escola de Engenharia de São Carlos, Universidade de São Paulo, São Carlos, 1997.

PALIWAL, R.; SHARMA, P.; KANSAL, A. Water quality modelling of the river Yamuna (India) using QUAL2E-UNCAS. Journal of Environmental Management, v. 83, n. 2, p. 131-144, 2007. http://dx.doi.org/10.1016/j.jenvman.2006.02.003 
COSTA, D. J. L.; TEIXEIRA, D. Análise de incerteza em um modelo matemático de qualidade da água aplicado ao Ribeirão do Ouro, Araraquara, SP, Brasil. Ambi-Agua, Taubaté, v. 6, n. 2, p. 232-245, 2011. (doi:10.4136/ambi-agua.196)

SÃO PAULO (Estado). Decreto $\mathbf{n}^{\circ} \mathbf{8 . 4 6 8}$ de 8 de setembro de 1976. Aprova o regulamento da Lei n. 997, de 31 de maio de 1976, que dispõe sobre a Prevenção e o Controle da Poluição do Meio Ambiente. 1976. Disponível em: <www.cetesb.sp.gov.br/Servicos/ licenciamento/postos/legislacao/Decreto_Estadual_8468_76.pdf $>$. Acesso em: 02 de abril de 2010.

SÃO PAULO (Estado). Decreto $\mathbf{n}^{\mathbf{0}} 10.755$ de 22 de novembro de 1977. Dispõe sobre o enquadramento dos corpos de água receptores na classificação prevista no Decreto $\mathrm{n}^{\mathrm{o}}$ 8.468, de 8 de setembro de 1976, e dá providências correlatas. 1977. Disponível em: $<$ www.cetesb.sp.gov.br/licenciamentoo/legislacao/estadual/decretos/1997_Dec_Est_10 755.pdf $>$. Acesso em: 02 de abril de 2010.

STREETER, H. W.; PHELPS, E. B. A study of the pollution and natural purification of the Ohio River. Public Health Bulletin, n. 146, p. 1-75, 1925.

TEIXEIRA, D.; RIOS, L.; COSTA, D. J. L.; FULLER, B. B.; SILVA L. N.; TEODORO, V. L. I. Mapeamento do uso e ocupação do solo e análise de um parâmetro indicador de qualidade da água da sub-bacia do Ribeirão do Ouro (Araraquara-SP). In: FÓRUM DE DESENVOLVIMENTO REGIONAL E MEIO AMBIENTE POLÍTICAS, LEGISLAÇÃO E GESTÃO AMBIENTAL, 2008, Araraquara. Anais... Araras: Uniara, 2008 .

VON SPERLING, M. Estudos e modelagem da qualidade da água de rios. Belo Horizonte: Departamento de Engenharia Sanitária e Ambiental; Universidade Federal de Minas Gerais, 2007. 